\title{
Spectral imaging of the retina
}

\begin{abstract}
Introduction The work described here involved the use of a modified fundus camera to obtain sequential hyperspectral images of the retina in 14 normal volunteers and in 1 illustrative patient with a retinal vascular occlusion.

Methods The paper describes analysis techniques, which allow oximetry within retinal vessels; these results are presented as retinal oximetry maps.

Results Using spectral images, with wavelengths between 556 and $650 \mathrm{~nm}$, the mean oxygen saturation (OS) value in temporal retinal arterioles in normal volunteers was $104.3( \pm 16.7)$, and in normal temporal retinal venules was $34.8( \pm 17.8)$. These values are comparable to those quoted in the literature, although, the venular saturations are slightly lower than those values found by other authors; explanations are offered for these differences.

Discussion The described imaging and analysis techniques produce a clinically useful map of retinal oximetric values. The results from normal volunteers and from one illustrative patient are presented. Further developments, including the recent development of a 'snapshot' spectral camera, promises enhanced non-invasive retinal vessel oximetry mapping.

Eye (2011) 25, 309-320; doi:10.1038/eye.2010.222
\end{abstract}

Keywords: spectral; hyperspectral; oximetry; retinal oximetry; retinal artery occlusion

\section{Introduction}

Spectroscopy is a well-established tool used in the analysis of a material through identification of the spectral signatures of its constituents. Spectral imaging extends the usefulness of spectroscopy by combining spectroscopy with imaging, thereby providing both spectral and spatial information. Spectral imaging requires
DJ Mordant' ${ }^{1}$, Al-Abboud², G Muyo², A Gorman², A Sallam¹, P Ritchie ${ }^{3}$, AR Harvey² and $\mathrm{Al}$ McNaught ${ }^{1,4}$

sensitive detectors and powerful computers to enable fast processing of images. Its use has expanded from remote sensing for both civilian and military purposes, to laboratory-based applications for biological imaging, such as cellular spectral imaging and oximetry. Spectral imaging systems record a stack of monochromatic images onto a two-dimensional detector array, such as a charge-coupled device (CCD); multiple images are collected over multiple wavelengths forming a 'spectral data cube'. Spectral imaging systems incorporate a range of strategies to enable collection of the multiple monochromatic images. There has been widespread application of spectral imaging systems in applications ranging from cytogenetics and ${ }^{1-4}$ pathology ${ }^{5}$ to oncology. ${ }^{6,7}$

The use of spectral imaging to perform blood oximetry, exploiting the different spectral characteristics of oxygenated $\left(\mathrm{HbO}_{2}\right)$ vs deoxygenated haemoglobin $(\mathrm{Hb})$ has been described in a variety of clinical applications, such as assessing tissue perfusion, ${ }^{8-10}$ microvascular disease in diabetes, ${ }^{11}$ and sickle cell anaemia. ${ }^{12}$

\section{Principles of blood oximetry}

The development of the photoelectric cell enabled the quantification of light absorption, which contributed to the development of the spectrophotometer. As a result, the spectrophotometric study of the extinction coefficients (a measurement of how much light is absorbed by a substance at a given wavelength) of reduced $\mathrm{Hb}$ and oxyhaemoglobin was made possible; these measurements are central to the technique of oximetry. The first studies of the spectra of undiluted haemolysed and whole blood were undertaken by Drabkin ${ }^{13,14}$, who demonstrated the applicability of the Lambert-Beer law in determining the OS of blood. The Lambert-Beer law, in transmission blood oximetry, assumes that for any given wavelength of light, its absorption is dependent on the extinction

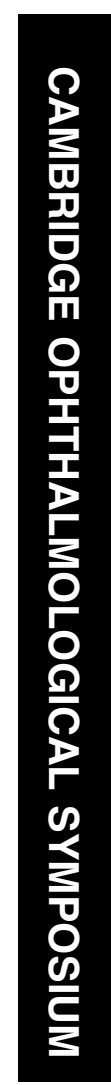

${ }^{1}$ Ophthalmology Department, Gloucestershire Eye Unit, Cheltenham General Hospital, Gloucestershire Hospitals NHS Foundation Trust, Cheltenham, UK

${ }^{2}$ School of Engineering and Physical Sciences, Heriot-Watt University, Edinburgh, UK

${ }^{3}$ Anaesthetics Department, Gloucestershire Hospitals NHS Foundation Trust, Cheltenham, UK

${ }^{4}$ Cranfield University, Bedfordshire, UK

Correspondence:

Al McNaught, Ophthalmology Department, Gloucestershire Eye Unit, Cheltenham General Hospital, Gloucestershire Hospitals NHS Foundation Trust Sandford Road, Cheltenham, Gloucestershire, GL53 7AN, UK.

Tel: + 01242272527 ;

Fax: +01242253816

E-mail: andy.mcnaught@ btopenworld.com

Received: 5 November 2010 Accepted in revised form: 22 November 2010 
coefficient of the blood solution $(\varepsilon)$, its concentration or haematocrit $(c)$, and the distance $(d)$ the light has to travel through the solution (path length):

$$
I_{T}=I_{o} 10^{-\varepsilon c d},
$$

where $I_{\mathrm{T}}$ is the intensity of light transmitted through a solution and $I_{\mathrm{o}}$ is the intensity of incident light.

The optical density (OD) of a solution is a measure of its attenuation, and is defined by:

$$
\mathrm{OD}=-\log _{10} \frac{I_{T}}{I_{0}}=\varepsilon c d
$$

Therefore, the OD of a solution of blood is a function of its extinction coefficient, concentration, and the path length the light has to travel. The main absorbing component of human blood is $\mathrm{Hb}$, therefore, its extinction coefficient is assumed to be equal to that of $\mathrm{Hb}$, although this is an approximation as it neglects scattering by the structure of red blood cells.

Kramer and Elam ${ }^{15}$ were able to demonstrate the linear relationship between the OS of blood and the absorption of red and infrared wavelengths of light. This finding was pivotal in the development of clinical oximeters. Matthes and Gross ${ }^{16,17}$ were the first to demonstrate the use of an isobestic wavelength and an oxygen-sensitive wavelength in the red and infrared regions to compensate for variables, such as the haematocrit, path length, and light intensity. This technique of measuring the blood OS using an isobestic and an oxygen-sensitive wavelength is the basis of clinical dual-wavelength oximetry used today. The integration of optical plethsmography (analysis of the pulsatile component of the arterial cycle) with oximetry enabled the development of pulse oximeters, which are capable of measuring the arterial OS by isolating the arterial signal from the venous, bone, and tissue components. ${ }^{18}$ The calibration of pulse oximeters is performed using data acquired from normal subjects during induced hypoxia. Unsurprisingly, these calibration tests were carried out at arterial blood gas saturations of greater than $70 \%$, the lowest tolerable state of systemic oxygenation in humans. At OSs below 70\%, the calibration has been estimated by linear interpolation, therefore, the accuracy below this level is questionable.

\section{Principles of retinal oximetry}

Retinal oximetry requires an imaging system, which captures images of the retina using multiple wavelengths of light. Image analysis of this spectral image allows the OD of the retinal vessels to be estimated at several discrete wavelengths. The OD at a retinal location is obtained by calculating the ratio of the measured

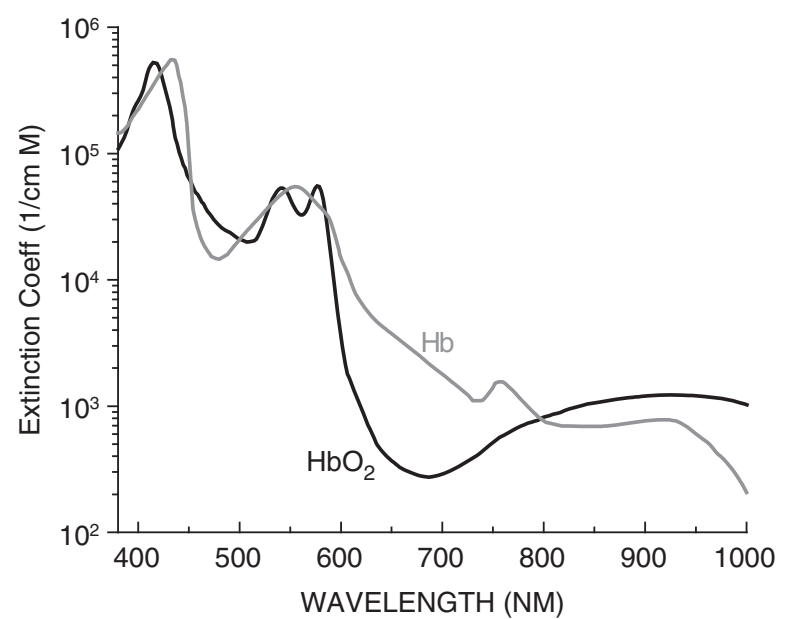

Figure 1 The molar extinction coefficients of deoxyhaemoglobin $(\mathrm{Hb})$ and oxyhaemoglobin $\left(\mathrm{HbO}_{2}\right)$ derivatives as a function of wavelength.

reflected light intensity adjacent to the target retinal vessel relative to the reflected light intensity at the centre of the retinal vessel:

$$
\mathrm{OD}_{\mathrm{vessel}}=-\log _{10} \frac{I_{V}}{I_{R}}
$$

where $I_{\mathrm{V}}$ and $I_{\mathrm{R}}$ are the intensity of light reflected from the retinal vessel and adjacent retina, respectively. The OD of the retinal vessels at various wavelengths can subsequently be used to calculate the OS (Figure 1).

Various approaches have been undertaken to non-invasively measure the OS of blood in retinal blood vessels. Gloster ${ }^{19}$ measured changes in choroidal reflectometry with altered oxygenation. Laing ${ }^{20}$ developed a 'choroidal eye oximeter' to measure the OS of choroidal blood. Hickam et $a l^{21}$ described a photographic method to estimate the OS of the retinal veins in humans. Delori et $a l^{22}$ described a method for determining the OS in retinal vessels using a three-wavelength photoelectric oximeter; this comprised a fundus camera integrated with three interference filters, a photomultiplier, and a photocathode. The accuracy of the OS calculations was reported to be most accurate in the range of $50-100 \%$ OS, but in the range between 0 and $50 \%$ there was a tendency towards overestimation of the OS. Delori et $a l^{23}$ later applied the three-wavelength oximetry technique in conjunction with laser Doppler velocimetry to study the effects of neurogenic optic atrophy with associated inner retinal degeneration on oxygen delivery to the retina. In eyes with optic atrophy, the mean venous OS was reported to be significantly higher in both temporal $(P=0.028)$ and nasal $(P=0.001)$ veins compared with the fellow eyes. 
Beach et $a l^{24}$ developed a retinal imaging system using two wavelengths of light to measure the OS in the retinal vasculature of diabetic subjects without clinical evidence of diabetic retinopathy during normoglycaemia and hyperglycaemia. Schweitzer et al developed an imaging ophthalmospectrometer, which consisted of a modified fundus camera with an attached spectrograph. The instrument illuminated the retina with a small $(40 \mu \mathrm{m} \times 1.5 \mathrm{~mm})$ slit of light. In vivo measurements of retinal vessel OS were performed using the oximetry algorithm in 30 eyes of 28 healthy Caucasian subjects. The mean OS in 126 retinal arteries and 139 retinal veins were $92.2 \pm 4.1$ and $57.9 \pm 9.9 \%$, respectively. The central retinal venous OS was higher in patients with age-related macular degeneration compared with the controls. ${ }^{25}$ A study on the OS in the retinal vessels of patients with primary open-angle glaucoma (POAG) using the imaging ophthalmospectrometer were performed by Michelson et al. Retinal arteriolar OS in eyes with normaltension POAG $(89.7 \pm 5.4 \%)$ was significantly lower than the control group $(P=0.006)$. Retinal venular OSs and arteriovenous differences in eyes with normal-tension POAG and high-tension POAG were not significantly different to the control eyes. ${ }^{26}$ The two-wavelength oximetry technique described by Beach et $a l^{24}$ was adopted by Stefánsson and co-workers who developed a retinal oximeter. Retinal vessel OSs of 16 normal eyes were compared at room air and during hyperoxia (100\% OS). Significant differences in the retinal vessel OSs were demonstrated. In the transition from normoxia to hyperoxia, the mean retinal arteriolar OS increased from $96 \pm 9$ to $101 \pm 8 \%(P=0.0027)$, and the retinal venules increased from $55 \pm 14$ to $78 \pm 15 \%(P<0.0001)$. This experiment demonstrated that the retinal oximeter was capable of detecting oximetric changes in the retinal vessels during hyperoxia. ${ }^{27} \mathrm{~A}$ similar dual wavelength retinal oximeter to that reported by Hammer et al used the same concept of retinal oximetry described by Beach et al. The retinal oximeter comprised of a fundus camera (Carl Zeiss Meditec, Jena, Germany) with an attached dual-wavelength filter, which enabled the transmission of light at wavelengths of $548 \pm 10$ and $610 \pm 10 \mathrm{~nm}$ (FWHM) to be recorded onto a colour CCD. The ODRs at the two wavelengths were used to calculate the OS. The mean $( \pm$ SD) arteriolar and venular OSs were $98 \pm 10.1$ and $65 \pm 11.7 \%$, respectively. Inhalation of $100 \%$ oxygen increased the mean arteriolar and venular OS by 2 and $7 \%$, respectively. The oximetry system was applied to study the OS in diabetic patients. Venular OS increased with the severity of diabetic retinopathy with a mean OS ( \pm SD) of $69 \pm 7 \%$ in subjects with mild non-proliferative DR and $75 \pm 8 \%$ in subjects with proliferative DR. ${ }^{28}$

The application of a Fourier transform spectral imaging system to quantify the oximetric status of the retina with ischaemia was reported in a study of human subjects with retinal vein occlusions. ${ }^{29}$ More recently, the Fourier transform spectral retinal imaging system was used to compare the OS of the retinal tissues and blood vessels around the optic disc in normal eyes, and eyes with open-angle glaucoma. ${ }^{30}$ Overall, 15 eyes with low-tension glaucoma and 41 eyes with hightension glaucoma were compared with 20 normal eyes. The average OS of the juxtapapillary retinal tissues in eyes with normal-tension glaucoma $(78 \pm 8 \%)$ and high-tension glaucoma $(82 \pm 10 \%)$ were reported to be significantly lower than in normal eyes $(88 \pm 8 \%$; $P=0.002$ and $P=0.039$, respectively).

\section{Materials and methods}

\section{Hyperspectral fundus camera}

The hyperspectral imaging system was built around a commercial mydriatic fundus camera (Canon CF-60Z, Tokyo, Japan), with a number of modifications to allow integration of a liquid crystal tunable filter (LCTF) into the optical path of the camera (Figure 2). This enabled illumination of the retina using a number of user-selected wavelengths of visible light. The LCTF chosen to be incorporated into the fundus camera was a CRI VariSpec (Woburn, MA, USA). The LCTF filter is able to transmit wavelengths between the visible and near-infrared region $(420-720 \mathrm{~nm})$ with a wavelength-dependent bandwidth of nominally $10 \mathrm{~nm}$. Importantly, the LCTF is electronically tunable, enabling fast random access of the desired wavelength via a personal computer, contains no moving parts, and has an operating ambient temperature range between 10 and $40^{\circ} \mathrm{C}$. The LCTF was integrated in an optimised position within the optical path of the incident light of the fundus camera. A low-noise CCD camera (Hamamatsu ORCA-ER, Hamamatsu Photonics KK, Hamamatsu City, Japan) was incorporated into the fundus camera to enable the electronic capture of retinal images. The CCD has a high resolution of approximately 1.3 million pixels and a dynamic range of 12 bits. The CCD is cooled to reduce dark current, noise, and thermal drift, and has a high-quantum efficiency in the visible and near-infrared wavelengths. A linear polariser is attached to the front of the CCD to reduce a fixed-pattern artifact caused by the specular reflection from the two surfaces of the objective lens within the fundus camera. Specular reflections from within the eye are also attenuated. A macro lens is inserted between the CCD and the fundus camera to maintain a field of view of approximately $40^{\circ}$ onto the CCD detector chip with dimensions of $6.6 \times 8.9 \mathrm{~mm}^{2}$ (Figure 2).

The hyperspectral imaging system is controlled with a customised software programmed using LabView 


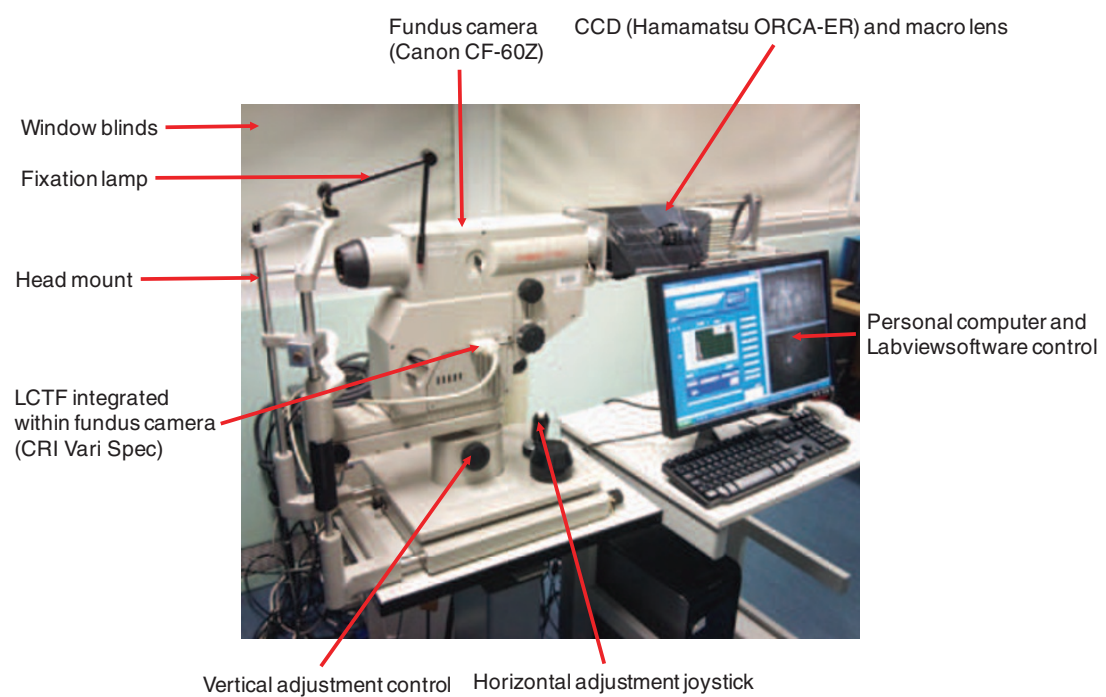

Figure 2 Incorporation of the CCD and LCTF into the fundus camera.

(National Instruments, Austin, TX, USA). This software was used to control the LCTF, trigger the xenon flash, and control the CCD camera to capture an image. The LCTF was controlled typically by changing the desired wavelengths in $10 \mathrm{~nm}$ increments or decrements. The xenon flash, engaged using the software, was integrated with the simultaneous capture of CCD images. A saturation histograph, live feed of the CCD recording, and image of a captured image were displayed on the software interface. The captured images were stored onto a personal computer in a 16-bit portable network graphics format.

\section{Human imaging and spectral image analysis}

We describe the use of hyperspectral fundus camera to acquire hyperspectral retinal images from healthy subjects and from an illustrative patient with a retinal branch artery occlusion, detailing the application of the image processing and analysis algorithms used to calculate the OS of the retinal vasculature. A total of 23 healthy subjects were recruited from the eye departments in Cheltenham and Gloucester General Hospitals. Written informed consent was obtained from all volunteers. All normal subjects were assessed by the study investigator. The best-corrected visual acuity (BCVA) was measured using a Snellen chart to record the distance vision. Intraocular pressures (IOPs) of both eyes were measured using Goldmann applanation tonometry. Slit-lamp biomicroscopy was used to assess the anterior segment. The pupils of the normal subjects were dilated with $1 \%$ tropicamide (Minims; Chauvin Pharmaceuticals, Romford, UK). Dilated fundoscopy was performed by slit-lamp biomicroscopy using a 78D lens. Oximetry measurements of the index finger were performed in all volunteers using a pulse oximeter
Table 1 Summarised clinical data of the normal subjects included in the study

\begin{tabular}{lc}
\hline Number & 14 \\
Mean age (range) (in years) & $42.9(25-74)$ \\
Mean best-corrected VA (LogMAR \pm SD) & $-0.07( \pm 0.09)$ \\
Mean intraocular pressure $(\mathrm{mm} \mathrm{Hg} \pm$ SD) & $14.1( \pm 2.0)$ \\
Mean systolic blood pressure $(\mathrm{mm} \mathrm{Hg} \pm$ SD) & $124.1( \pm 10.1)$ \\
Mean diastolic blood pressure $(\mathrm{mm} \mathrm{Hg} \pm$ SD) & $78.5( \pm 10.4)$ \\
Mean pulse oximeter oxygen saturation $(\% \pm \mathrm{SD})$ & $96.7( \pm 0.8)$ \\
\hline
\end{tabular}

(Biox 3740, Ohmeda, Louisville, CO, USA). Brachial blood pressure of all normal subjects was measured using an aneroid sphygmomanometer (Accoson 0342, AC Cossor \& Son (Surgical) Ltd., Essex, UK).

Inclusion criteria were BCVA 6/9 or better, open anterior-chamber angle, absence of any ocular pathology, IOP $<21 \mathrm{~mm} \mathrm{Hg}$, and normal optic-disc appearance. Exclusion criteria included history of intraocular surgery, ocular pathology, diabetes mellitus, stroke, hypertension, and glaucoma family history. The clinical details of the included normal subjects are summarised in Table 1.

\section{Acquisition of hyperspectral retinal images}

Spectral images of the retina were acquired using wavelengths between 500 and $650 \mathrm{~nm}$ at $2 \mathrm{~nm}$ intervals. Eye fixation was maintained by instructing the subject to fixate on a red-fixation light with the contralateral eye. The average time to acquire a full data set of spectral images was approximately $10-15 \mathrm{~min}$. Retinal images were obtained from 23 normal volunteers and from one patient with a branch retinal artery occlusion. 


\section{Results I}

\section{Qualitative features of the retinal blood vessels in spectral images of the retina}

Spectral images of the retina revealed characteristic features of the retinal blood vessels. First, the retinal arterioles (Figure 3, red arrow heads) become less optically dense compared with the retinal venules

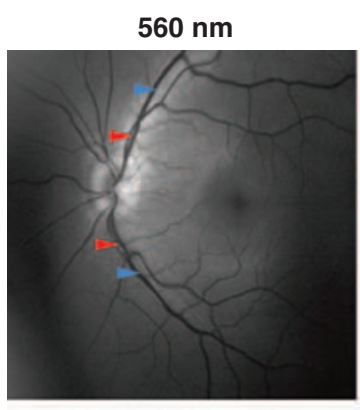

$580 \mathrm{~nm}$

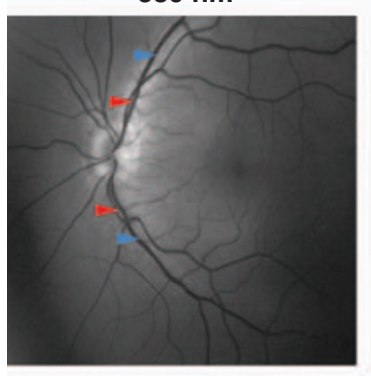

$600 \mathrm{~nm}$

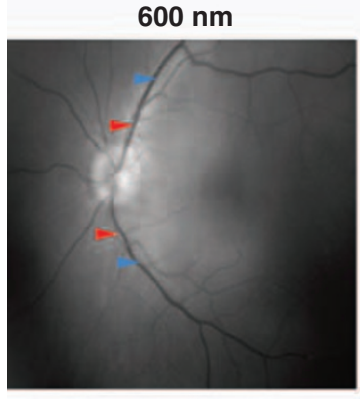

$620 \mathrm{~nm}$

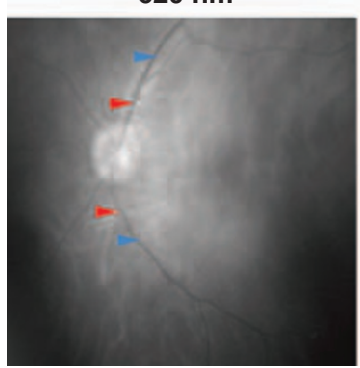

$570 \mathrm{~nm}$

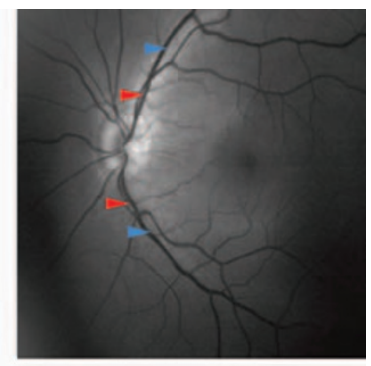

$590 \mathrm{~nm}$

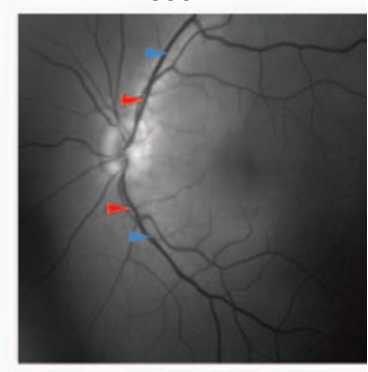

$610 \mathrm{~nm}$

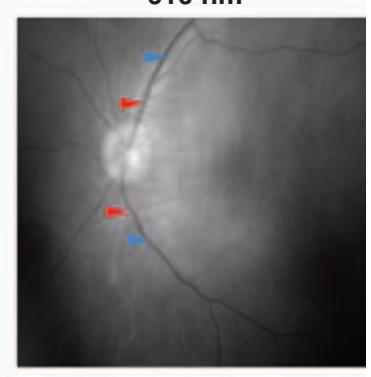

$630 \mathrm{~nm}$

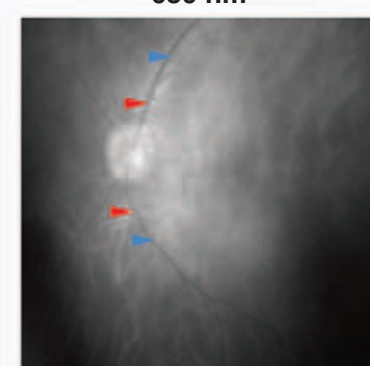

Figure 3 The appearances of the arterioles and venules at nine selected wavelengths in the dark calibrated images of the retina in a normal subject. At wavelengths $>600 \mathrm{~nm}$, arterioles (red arrows) are less optically dense compared with the venules (blue arrows).
(Figure 3, blue arrow heads) at wavelengths greater than $590 \mathrm{~nm}$. This feature is consistent with the respective extinction coefficients of $\mathrm{HbO}_{2}$ and deoxyhaemoglobin; the $\mathrm{OD}$ of $\mathrm{HbO}_{2}$ is lower than that of deoxyhaemoglobin between 600 and $650 \mathrm{~nm}$. Second, the macular branches of the retinal venules also appear to be less optically dense than the first-degree retinal venules, suggesting that the macular branches of the retinal venules appear to have relatively higher OSs than the first-degree venular vessels.

\section{Results II}

\section{Processing of hyperspectral retinal images}

The specific image processing algorithms that were applied to the retinal images are described in the following sections:

\section{Image registration}

The image registration method used a cross-correlation technique, which measures the degree of similarity between two images. A reference image is chosen, usually at $580 \mathrm{~nm}$, in which the OD of the arterioles and venules are similar, and both demonstrate relatively high contrast relative to the retinal background. All of the other spectral retinal images are aligned to the coordinates of the reference image. The maximum cross-correlation within each image is searched for by rotating and translating the images, and comparing with the reference image. The cross-correlation techniques require features within each image to be similar in appearance, irrespective of the wavelength. However, the appearance of the spectral retinal images change with wavelength (Figure 3). Hence, a number of preprocessing steps were implemented during the image registration process. First, sequential spectral retinal images were acquired such that the main features of the retinal images (optic disc and large retinal vessels) were located in approximately at the same position in the image field. Second, the vascular network and the optic disc were used as features within each image, and enhanced to enable accurate cross-correlation between the reference retinal image and the sequential spectral images. These features were enhanced using customised spatialfrequency passband and edge detection (Laplacian or Gaussian) filters. Some image series could not be co-registered because of poor image quality, poor focus, and/or excessive movement between individual spectral images in the series; these image series were discarded.

\section{Vessel detection and tracking}

The coordinates of the centreline of the retinal vasculature were calculated by manually detecting the 
retinal blood vessels and applying a semi-automated vessel-tracking routine. The vessel-tracking technique is based on a Fast-marching algorithm, which searches for pixels between two points, with the lowest intensities, and finds the shortest path. ${ }^{31}$ To enable accurate tracking of the retinal vasculature, a reference retinal image $(580 \mathrm{~nm})$ was converted into an image, which highlighted the retinal blood vessels by assigning the lowest pixel values to the vessels in contrast with higher pixel values to the retinal background. Two points along the length of a given blood vessel segment within the reference image were then manually selected, and the vessel-tracking algorithm was applied. This process was repeated until the majority of the retinal vasculature was tracked. Accurate tracking for small arterioles and venules were often the most difficult to obtain, and therefore tracking of some of the smaller blood vessels were omitted because of vessel-tracking errors. The vessel-tracking algorithm enabled an estimate of the centreline coordinates of the retinal blood vessels.

Vessel profile extraction and calculation of the light transmission of the retinal vasculature

The centreline coordinates along each blood vessel were subsequently used to calculate the coordinates of a series of linear profiles orthogonal to the vessels. This was performed by calculating the perpendicular of the gradient between two successive points along the centre of a given blood vessel. The result is a series of linear profiles perpendicular to the blood vessels in the retinal vascular network (Figure 4). The length of the profile was manually selected depending on the size of the blood vessel, and was approximately 2-3 times the estimated width of the blood vessel. The intensity values along the length of each linear profile were extracted.
This was repeated for all the spectral images such that for each linear profile in a given blood vessel, the intensity values along that profile were extracted for all wavelengths.

\section{Optical density estimation}

Fitting algorithms were applied to each gray-scale intensity profile to estimate the intensity at the centre of the blood vessel (minima of the nonlinear curve) and intensity of the background adjacent to the blood vessel (gray-scale value of the linear fit at the centre of the blood vessel). The optical densities of a given point were estimated across the wavelength range $(500-650 \mathrm{~nm})$ to calculate the OD profile.

This was repeated at all points along all the selected blood vessels.

\section{Oxygen saturation calculation}

This was performed by using an algorithm based on the Levenberg-Marquardt nonlinear fit to the complete set of OD $(\lambda)$ for a given point on the tracked blood vessel in the equation,

$$
\begin{aligned}
O D(\lambda)= & a S(\lambda)+\eta(\lambda) C_{H b \text { Total }} d\left(\varepsilon_{o x y}(\lambda)-\varepsilon_{d e-o x y}(\lambda)\right) c O S \\
& \left.+\varepsilon_{d e-o x y}(\lambda)\right),
\end{aligned}
$$

where, $C_{\mathrm{Hb} \text { total }}$ is the total concentration of $\mathrm{Hb}, \varepsilon_{\mathrm{oxy}}$ and $\varepsilon_{\text {de-oxy }}$ are the extinction coefficients of $\mathrm{HbO}_{2}$ and deoxygenated $\mathrm{Hb}$, respectively, corrected for convolution with the spectral response of the LCTF; $d$ is the vessel diameter, $c O S$ is the calculated OS, $\eta$ is the effective optical path-length contribution, ${ }^{38}$ and $a$ is a scaling constant. The function accounts for the backscatter by blood cells. The algorithm estimates the free parameters $\left(C_{\mathrm{Hb} \text { Total }}, d, \eta, a, S\right)$ to provide an estimate of $c O S$. Oxygen saturation calculations were performed at all points
Arterioles

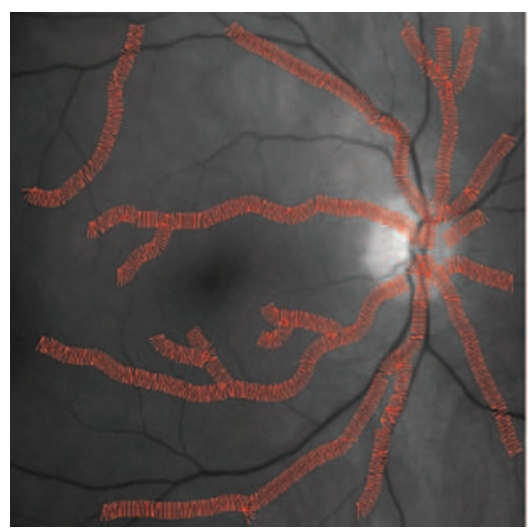

Venules

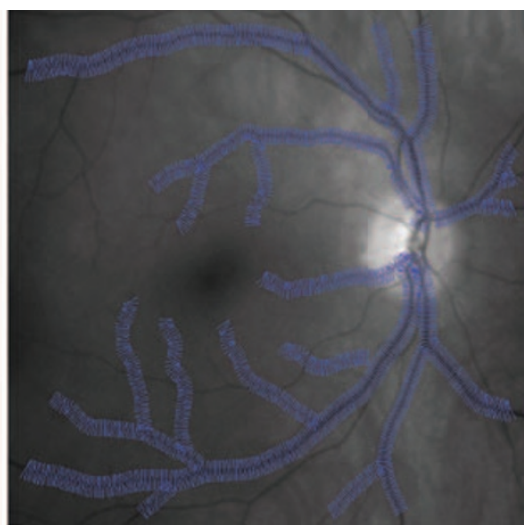

Figure 4 Linear profiles perpendicular to the retinal blood vessels in an image of a normal right-eye retina. 
along the tracked centreline of selected retinal blood vessels. Optimal OS results were attained when the spectral images between 556 and $640 \mathrm{~nm}$ were analysed. The OS calculations and corresponding coordinates of the centre of the tracked retinal blood vessels were used to generate oximetric pseudocolour maps of the retinal vasculature.

To enable further studies of the OS data, a semi-automated program was written in Mathematica (ver. 5.2; Wolfram Research, Inc., Champaign, IL, USA) to enable the extraction of calculated OS values at given points in the retinal arterioles and venules defined by the distance from the optic-disc margin. This was performed by determining the centre of the optic disc and its radius $(r)$. Four additional circles with increasing radii $(2 r, 3 r, 4 r, 5 r)$ about the point in the centre of the optic disc corresponded to the regions $\frac{1}{2}$ disc diameter (dd), $1 \mathrm{dd}, 1 \frac{1}{2} \mathrm{dd}$ and $2 \mathrm{dd}$ from the disc margin, respectively.

\section{Oximetric maps of the retinal vasculature in normal subjects}

Figure 5 shows pseudocolour images of the calculated OSs along the retinal arterioles and venules in three example normal subjects. It can be observed that the retinal arterioles generally have calculated OS between 70 and $100 \%$. The calculated OSs of the retinal venules appear to be more varied. The mean OS calculation $( \pm S D)$ of the temporal retinal arterioles and venules using a wavelength sequence between 556 and $650 \mathrm{~nm}$ were $104.3( \pm 16.7)$ and $34.8( \pm 17.8) \%$, respectively.
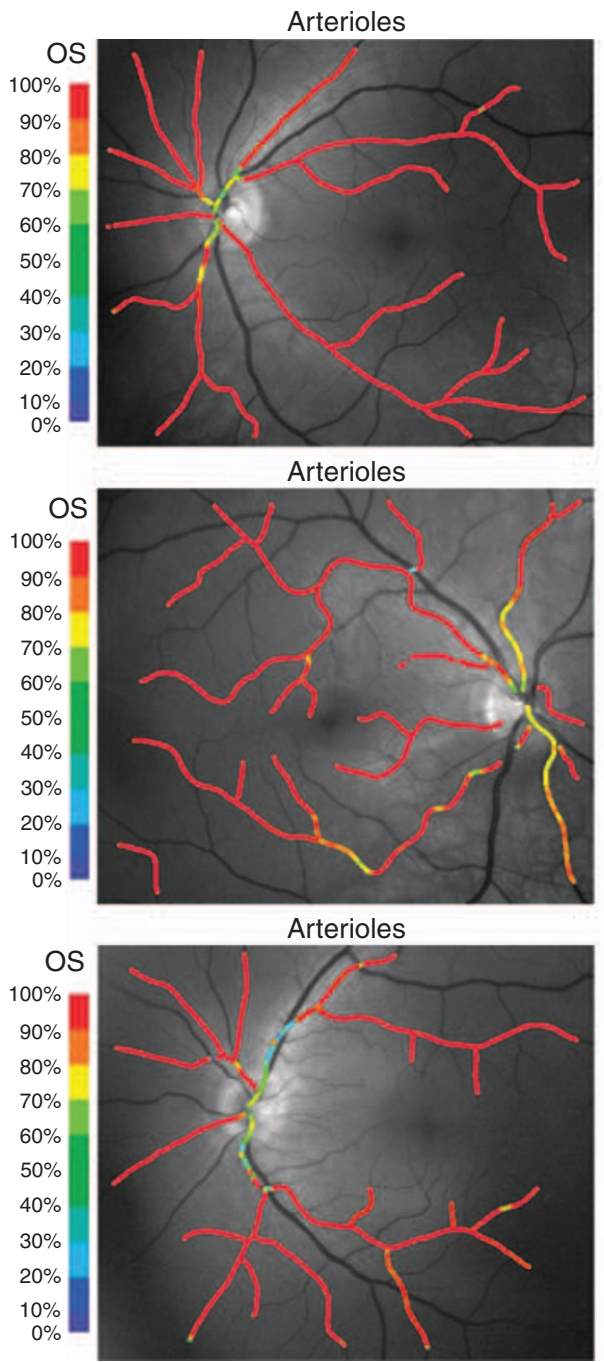
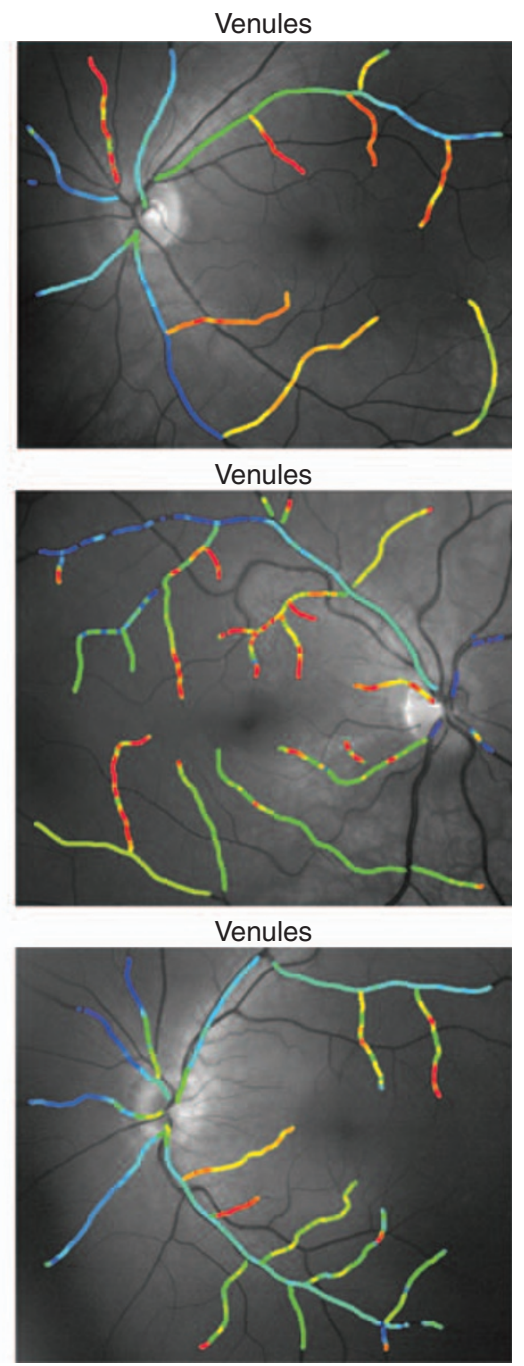

Figure 5 Pseudocolour images of OS maps superimposed on the retinal arterioles (left) and retinal venules (right) in three normal subjects. 
The macular branches of the retinal venules generally appear to have higher calculated OSs compared with their respective main trunk first and second-degree retinal venules. Figure 6 shows box- and whisker plots of the OS of the (main trunk) temporal retinal arterioles and venules in normal subjects, with the median, range, and the minimum and maximum values displayed.

These values changed very little with increasing eccentricity from the optic-disc margin. Figure 7 shows that the mean OS (and SEM) of the temporal retinal arterioles and venules at different (at the disc margin, $\frac{1}{2} \mathrm{dd}, 1 \mathrm{dd}, 1 \frac{1}{2} \mathrm{dd}$ and $2 \mathrm{dd}$ ) eccentricities from the optic-disc margin were not associated with significant

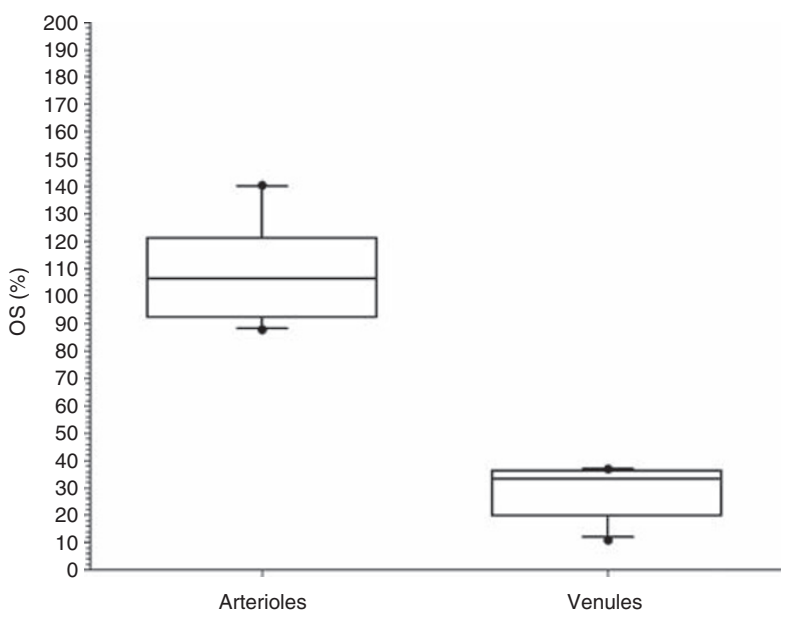

Figure 6 A box-and-whisker plot of the OS of the temporal (superotemporal and inferotemporal) retinal arterioles and venules in normal subjects. The plots show the median, the interquartile range, 95\% central range, and the minimum and maximum values. changes in the OS; temporal retinal arterioles $r^{2}=0.071$, $P=0.66$, and venules $r^{2}=0.002, P=0.95$, respectively.

\section{Results III}

\section{Example of retinal vascular disease: branch retinal artery occlusion}

Patient 1 was a 79-year-old man with a 25-day history of a left-inferior branch retinal artery occlusion (Figure 8a). The VA of the left eye was 6/18 at the time of imaging. The pseudocolour images of the OS calculations overlayed onto the retinal vessels (Figure $8 \mathrm{~b}$ ) indicate lower than normal OSs of the inferotemporal retinal arterioles, corresponding to the location of occluded retinal arteriole.

\section{Discussion}

The human retina has a dual blood supply to meet the high metabolic demands of the photoreceptors, ganglion cells, and the retinal pigment epithelium. The inner two-thirds of the retina is supplied from branches of the central retinal vessels, and the outer one-third of the retina is supplied by the choroid creating a watershed zone at the outer plexiform layer. The retinal circulation is characterised by a low blood flow rate $(80 \mathrm{ml} / \mathrm{min})$, but the choroid, in contrast, has a high level of flow $(800 \mathrm{ml} / \mathrm{min}){ }^{32,33}$ Choroidal arterio-venous OS difference is 3 vs (at least) $40 \%$ for the retinal circulation. ${ }^{34}$ This higher OS differences between arterial and venous blood in the retinal circulation offers the possibility of gaining insights into inner retinal health by the measurement of retinal vessel OS, and quantification of changes in arterio-venous OS, with physiological
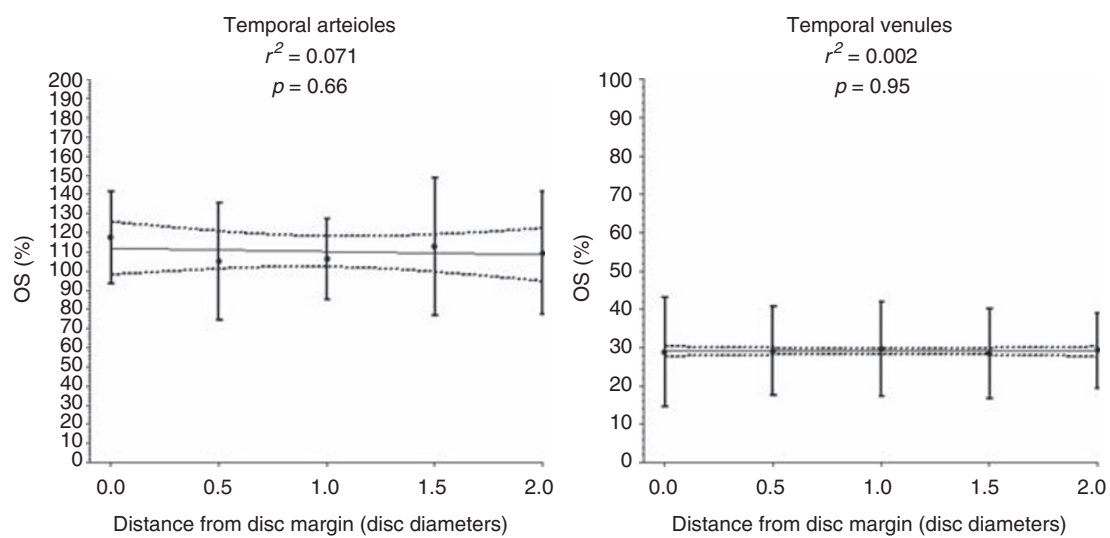

Figure 7 Oxygen saturation calculations of the temporal (superotemporal and inferotemporal) arterioles (left) and venules (right) at points defined by the distance from the optic disc: at the disc margin (0.0), $\frac{1}{2}$ disc diameter (0.5), $1 \mathrm{dd}(1.0), 1 \frac{1}{2} \mathrm{dd}(1.5)$ and $2 \mathrm{dd}(2.0)$ from the disc margin. The mean OS (central black filled circle), the SEM (vertical lines), the best-fit line (solid black horizontal line), and the $95 \%$ confidence interval of the best-fit line (horizontal dashed lines) are shown in the plots. 
a
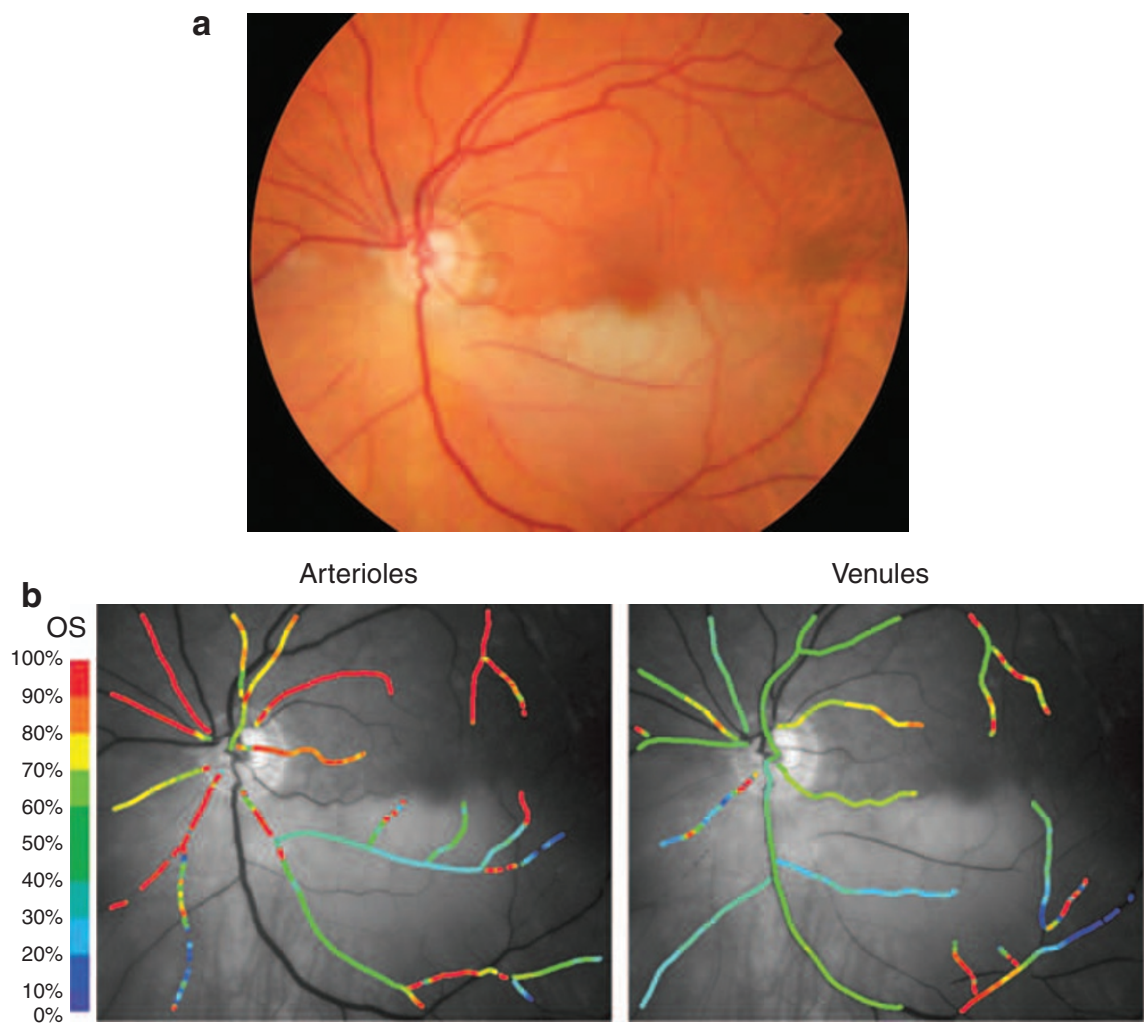

Figure 8 (a) Colour fundus photograph of patient 1, showing pale inferotemporal retina, corresponding to region affected by inferotemporal retinal arteriole occlusion. (b) Pseudocolour oximetry map showing abnormally low OS within the affected inferotemporal retinal arteriole in contrast to the normal OS levels in the unaffected superotemporal arteriole. The corresponding inferotemporal retinal venule has a normal level of OS.

changes, for example, dark adaption and/or in response to retinal disease.

The accurate quantification of retinal oxygen concentration in retinal tissues, and the OS in retinal blood vessels, therefore, remains an important but challenging, goal. A number of different approaches have been reported in the literature. A significant proportion of retinal oxygenation studies have been focused on the use of oxygen-sensitive microelectrodes in studying the $\mathrm{O}_{2}$ tension $\left(\mathrm{pO}_{2}\right)$ in the retina. Importantly, this technique is able to measure the $\mathrm{pO}_{2}$ gradients across the choroid, retina, and vitreous, which provide insights into the local metabolic state of the retina. Birol et $a l^{35}$ studied the distribution of foveal and perifoveal oxygen in six anaesthetised cynomolgus macaques using $\mathrm{O}_{2}$ microelectrodes. Two characteristic $\mathrm{pO}_{2}$ minima were identified in the fovea and perifovea at the level of the photoreceptor inner segments and at the vitreo-retinal interface. The invasiveness of this technique has restricted its use mainly in animal studies. ${ }^{36}$ Studies in human eyes have been restricted to measurements of vitreous $\mathrm{pO}_{2}$ during intraocular surgery. ${ }^{37-39}$

The potential value of the clinical measurement, but the invasive nature of the techniques described above, have ensured that non-invasive retinal vessel oximetry in humans remains an important aspiration. This report describes the design of a retinal camera, which has allowed successful acquisition of spectal images of the retina, and details the image processing and analysis techniques, which have been used to calculate the OS of the retinal vasculature in a small group of normal subjects and in an illustrative example of a patient with a branch retinal arterial occlusion. We have previously reported the validation of this spectral imaging technique using a model eye with artificial retinal vessels filled with human blood of known OS. ${ }^{40}$ A wavelength sequence of $556-650 \mathrm{~nm}$ was determined to be the most appropriate wavelength sequence to accurately calculate the OS of the retinal vasculature. This wavelength sequence incorporates the important features of the extinction coefficient curves of $\mathrm{HbO}_{2}$ (local minima and maxima) and deoxyhaemoglobin (local maxima) to enable the nonlinear fitting oximetry algorithm to accurately differentiate most OD profiles of blood with varying OSs.

A total of 14 normal volunteers were successfully imaged, and retinal oximetry values were obtained. Examples of the pseudocolour images of OS calculations, overlayed onto the retinal arterioles and venules in three 
example normal subjects are illustrated in Figure 5. Lower than expected OSs of the retinal arterioles can be observed frequently at locations where the arterioles are in close proximity to the larger venules. These errors are mostly due to inaccuracies of the vessel profile analysis; to analyse the light transmission of a vessel, linear profiles orthogonal to the tracked coordinates of the vessels are generated. The vessel profiles of tracked arterioles adjacent to the larger venules will crossover onto the spatial coordinates of the venules, resulting in the accidental 'contamination' of the OD calculation related to the arteriole compared with that associated with the venule (lower OS). Careful selection of appropriate points along the arterioles, at given distances from the optic-disc margin, was performed to reduce this tendency. Furthermore, OS calculations of small segments of the vessels were obtained for the segmentation analysis as opposed to large segments of the vessels to reduce the influence of spurious outlying results. Notwithstanding these precautions during analysis, physical trans-luminal diffusion of oxygen down its concentration gradient, from the arteriole to the venule, cannot be excluded as a potential additional factor. The cause of the variability of the OS in the retinal venules is unclear, and could be attributed to a number of factors, including variability in the illumination of the retina images (vignetting) and retinal background (variable pigmentation). Additionally, it could be explained by physiological variability in the function of the retina in the photopic conditions used to acquire the spectral retinal images, variable consumption of oxygen, and short-term changes in retinal blood flow. Analysis of the first- and second-degree temporal retinal arterioles at defined eccentricity from the optic-disc margin revealed a mean OS of $110.8( \pm 11.8) \%$. This is comparable to OS measurements of retinal arterioles in healthy subjects reported by previous authors.

Correlation and linear regression analyses indicated that the OS of the first- and second-degree temporal retinal arterioles did not significantly change at varying eccentricity from the optic-disc margin of up to $2 \mathrm{dd}$. However, the mean OS of the first- and second-degree temporal retinal venules was $27.7( \pm 19.1) \%$, which is lower than the OS measurements of the retinal venules reported in previous studies. A possible explanation could be an error in the OS calculation caused by inaccurate curve fitting by the nonlinear algorithm to the OD profiles. However, this is unlikely as all the OD profiles included in the analysis were carefully selected and examined. Poor quality OD profiles were excluded from the analysis. The constant OS measurements of the retinal venules at different eccentricities from the opticdisc margin indicate consistency in the OS measurements. Previous retinal oximetry systems have reported retinal venous OSs ranging from 45 to $65 \%$. Most of these systems rely on two wavelength oximetry, which have been reported to overestimate the true OS.34 A further possible explanation could be the wavelengths used by other workers, ${ }^{25}$ which ranged between 510 and $586 \mathrm{~nm}$ in 2-nm increments. At these wavelengths, the hyperspectral retinal imaging system described in this present study provided highly variable optical densities of the retinal vessels, which could possibly contribute to errors in the OS calculations.

The research work described has a number of limitations. Data is presented from a relatively small number of normal subjects. Data from nine normal subjects $(40 \%)$ had to be excluded from the study because of poor image quality, resulting in poor OD calculations. Unfortunately this was unavoidable, as the current image acquisition process and subsequent analysis is cumbersome and demanding for both the volunteer and the operator. Acquisition and processing of the images to generate oximetry maps of the retinal vasculature of one eye took on average approximately $50 \mathrm{~h}$ of cumulative processing on a computer with a $2.5 \mathrm{GHz}$ dual-core processor with $4 \mathrm{~Gb}$ of RAM. The processing and analysis steps are semi-automated, and in addition to this, further analysis had to be performed to calculate the OSs of the retinal vessels at defined points along the retinal vessels to give the mean OSs of the temporal retinal arterioles and venules. Furthermore, inaccuracies of the OD and OS calculations were frequently encountered in regions of the retinal images in which there was poor illumination of the retina, particularly towards the periphal nasal retina: calculated OSs beyond the physiological range (0-100\%), negative values and some calculated OSs greater than $100 \%$. As a result, analysis of segments of the retinal vasculature was limited to $2 \mathrm{dd}$, and analysis of the nasal retinal vasculature was not performed. In this study, truncation of the analysed wavelength sequence of available optical densities before oxygen calculations improved oximetry accuracy. The underlying cause of variability of the optical densities at wavelengths less than $556 \mathrm{~nm}$ is unclear. At these wavelengths, the retina appears dark in the spectral images. The reduced contrast between the retinal background and retinal vessels could be a plausible explanation for the variability in the $\mathrm{OD}$ calculations at these wavelengths. Although reducing the wavelength sequence has reduced the effects of this variability, the wavelengths between 556 and $650 \mathrm{~nm}$ are possibly still susceptible to variations in the retinal pigmentation. This was noticeable in the analysis of two normal subjects with highly pigmented retinas.

In summary, this study has described techniques to deliver retinal vessel OS calculations in normal subjects 
and in a retinal patient. Retinal arteriolar OS values were consistent with previous studies. Retinal venular OS values were lower than some reports on normal subjects in the literature, however, the values were comparable to those reported by Delori et al. ${ }^{22,23}$ Retinal vessel oximetry using the described imaging and analysis techniques, especially using recently developed 'snapshot' spectral imaging refinements ${ }^{41}$ holds promise for accurate, practical, and non-invasive retinal oximetry measurements.

\section{Conflict of interest}

AR Harvey owns a patent to a related spectral imaging device, and rest of the authors declare no conflict of interest.

\section{Acknowledgements}

This study was supported by Eye Therapy Trust; Technology Strategy Board (CHBT/007/00028). This paper presents independent research awarded under New and Emerging Applications of Technology (NEAT), part of the i4i Invention for Innovation programme (NEAT K034) of the National Institute for Health Research (NIHR). The views expressed in this publication are those of the author(s) and not necessarily those of the NHS, the NIHR, or the Department of Health. This study was approved by the Gloucestershire Research Ethics Committee (COREC reference: 06/Q2005/131) and all procedures were carried out in accordance with the tenets of the Declaration of Helsinki.

\section{References}

1 Nuffer LL, Medvick PA, Foote HP, Solinsky JC. Multispectral/hyperspectral image enhancement for biological cell analysis. Cytometry A 2006; 69(8): 897-903.

2 Timlin JA, Haaland DM, Sinclair MB, Aragon AD, Martinez MJ, Werner-Washburne M. Hyperspectral microarray scanning: impact on the accuracy and reliability of gene expression data. BMC Genomics 2005; 6(1): 72 .

3 Huebschman ML, Schultz RA, Garner HR. Characteristics and capabilities of the hyperspectral imaging microscope. IEEE Eng Med Biol Mag 2002; 21(4): 104-117.

4 Schultz RA, Nielsen T, Zavaleta JR, Ruch R, Wyatt R, Garner HR. Hyperspectral imaging: a novel approach for microscopic analysis. Cytometry 2001; 43(4): 239-247.

5 Rooney PJ. Rapid identification of urinary tract infection bacteria using hyperspectral whole-organism fingerprinting and artificial neural networks. Microbiology 1998; 144(5): 1157-1170.

6 Martin ME, Wabuyele MB, Chen K, Kasili P, Panjehpour M, Phan $\mathrm{M}$ et al. Development of an advanced hyperspectral imaging (HSI) system with applications for cancer detection. Ann Biomed Eng 2006; 34(6): 1061-1068.
7 Sorg BS, Moeller BJ, Donovan O, Cao Y, Dewhirst MW. Hyperspectral imaging of hemoglobin saturation in tumor microvasculature and tumor hypoxia development. J Biomed Opt 2005; 10(4): 44004.

8 Cancio LC, Batchinsky AI, Mansfield JR, Panasyuk S, Hetz K, Martini D et al. Hyperspectral imaging: a new approach to the diagnosis of hemorrhagic shock. J Trauma 2006; 60(5): 1087-1095.

9 Zuzak KJ, Schaeberle MD, Lewis EN, Levin IW. Visible reflectance hyperspectral imaging: characterization of a noninvasive, in vivo system for determining tissue perfusion. Anal Chem 2002; 74(9): 2021-2028.

10 Zuzak KJ, Schaeberle MD, Gladwin MT, Cannon III RO, Levin IW. Noninvasive determination of spatially resolved and time-resolved tissue perfusion in humans during nitric oxide inhibition and inhalation by use of a visible-reflectance hyperspectral imaging technique. Circulation 2001; 104(24): 2905-2910.

11 Greenman RL, Panasyuk S, Wang X, Lyons TE, Dinh T, Longoria L et al. Early changes in the skin microcirculation and muscle metabolism of the diabetic foot. Lancet 2005; 366(9498): 1711-1717.

12 Zuzak KJ, Gladwin MT, Cannon III RO, Levin IW. Imaging hemoglobin oxygen saturation in sickle cell disease patients using noninvasive visible reflectance hyperspectral techniques: effects of nitric oxide. Am J Physiol Heart Circ Physiol 2003; 285(3): H1183-H1189.

13 Drabkin DL, Austin JH. Spectrophotometric studies. V. Technique for analysis of undiluted blood and concentrated hemoglobin solutions. J Biol Chem 1935; 112: 105-115.

14 Drabkin DL, Schmidt CF. Observations of circulating blood in vivo, and the direct determination of the saturation of hemoglobin in arterial blood. J Biol Chem 1945; 157: 69-83.

15 Kramer K, Elam JO, Saxton GA, Elam Jr WN. Influence of oxygen saturation, erythrocyte concentration and optical depth upon the red and near-infrared light transmittance of whole blood. Am J Physiol 1951; 165: 229-246.

16 Matthes K, Gross F. Untersuchungen fiber die absorption yon rotem und ultraotem Licht durch kohlenoxydgesittigtes und reduziertes Blut. Arch Exp Pathol Pharmacol 1939; 191: 369-380.

17 Matthes K, Gross F. Fortlaufende Registrierung der Lichtabsorption des Blutes in zwei verschiedenen pektralbezirken. Arch Exp Pathol Pharmacol 1939; 191: 381-390.

18 Aoyagi T, Kishi M, Yamaguchi K, Watanabe S. Improvement of the earpiece oximeter. Abstracts of the 13th annual meeting of the Japanese Society of Medical Electronics and Biological Engineering 1974; 90-91.

19 Broadfoot KD, Gloster J, Greaves DP. Photoelectric method of investigating the amount and oxygenation of blood in the fundus oculi. Br J Ophthalmol 1961; 45(3): 161-182.

20 Laing RA, Danisch LA, Young LR. The choroidal eye oximeter: an instrument for measuring oxygen saturation of choroidal blood in vivo. IEEE Trans Biomed Eng 1975; 22(3): 183-195.

21 Hickam JB, Frayser R, Ross JC. A study of retinal venous blood oxygen saturation in human subjects by photographic means. Circulation 1963; 27: 375-385.

22 Delori FC. Noninvasive technique for oximetry of blood in retinal vessels. Applied Optics 1988; 27(6): 1113-1125.

23 Sebag J, Delori FC, Feke GT, Weiter JJ. Effects of optic atrophy on retinal blood flow and oxygen saturation in humans. Arch Ophthalmol 1989; 107(2): 222-226. 
24 Tiedeman JS, Kirk SE, Srinivas S, Beach JM. Retinal oxygen consumption during hyperglycemia in patients with diabetes without retinopathy. Ophthalmology 1998; 105(1): 31-36.

25 Schweitzer D, Hammer M, Kraft J, Thamm E, Königsdörffer E, Strobel J. In vivo measurement of the oxygen saturation of retinal vessels in healthy volunteers. IEEE Trans Biomed Eng 1999; 46(12): 1454-1465.

26 Michelson G, Scibor M. Intravascular oxygen saturation in retinal vessels in normal subjects and open-angle glaucoma subjects. Acta Ophthalmol Scand 2006; 84: 89-295.

27 Hardarson SH, Harris A, Karlsson RA, Halldorsson GH, Kagemann L, Rechtman E et al. Automatic retinal oximetry. Invest Ophthalmol Vis Sci 2006; 47(11): 5011-5016.

28 Hammer M, Thamm E, Schweitzer D. A simple algorithm for in-vivo ocular fundus oximetry compensating for nonhaemoglobin absorption and scattering. Phys Med Biol 2002; 47(17): N233-N238.

29 Yoneya S, Saito T, Nishiyama Y, Deguchi T, Takasu M, Gil T et al. Retinal oxygen saturation levels in patients with central retinal vein occlusion. Ophthalmology 2002; 109(8): 1521-1526.

30 Ito M, Murayama K, Deguchi T, Takasu M, Gil T, Araie M et al. Oxygen saturation levels in the juxta-papillary retina in eyes with glaucoma. Exp Eye Res 2008; 86(3): 512-518.

31 Sethian JA. Level Set Methods and Fast Marching Methods. Cambridge University Press: Cambridge, Massachusetts, 1999.

32 Shimada Y, Yoshiya I, Oka N, Hamaguri K. Effects of multiple scattering and peripheral circulation on arterial oxygen saturation measured with a pulse-type oximeter. Med Biol Eng Comput 1984; 22: 475-478.

33 Alm A, Bill A. Ocular and optic nerve blood flow at normal and increased intraocular pressures in monkeys (Macaca irus): a study with radioactively labelled microspheres including flow determinations in brain and some other tissues. Exp Eye Res 1973; 15(1): 15-29.

34 Feke GT, Tagawa H, Deupree DM, Goger DG, Sebag J, Weiter JJ. Blood flow in the normal human retina. Invest Ophthalmol Vis Sci 1989; 30(1): 58-65.

35 Törnquist $\mathrm{P}$, Alm A. Retinal and choroidal contribution to retinal metabolism in vivo. A study in pigs. Acta Physiol Scand 1979; 106(3): 351-357.

36 Birol G, Wang S, Budzynski E, Wangsa-Wirawan ND, Linsenmeier RA. Oxygen distribution and consumption in the macaque retina. Am J Physiol Heart Circ Physiol 2007; 293(3): H1696-H1704.

37 Sakauke H, Negi A, Honda Y. Comparative study of vitreous oxygen tension in human and rabbit eyes. Invest Ophthalmol Vis Sci 1989; 30: 1933-1937.

38 Stefansson E, Machemer R, de Juan E, McCuen II BW. Retinal oxygenation and laser treatment in patients with diabetic retinopathy. Am J Ophthalmol 1992; 113: 36-38.

39 Williamson TH, Grewal J, Gupta B, Mokete B, Lim M, Fry $\mathrm{CH}$. Measurement of $\mathrm{PO}_{2}$ during vitrectomy for central retinal vein occlusion, a pilot study. Graefes Arch Clin Exp Ophthalmol 2009; 247(8): 1019-1023.

40 Mordant DJ, Al-Abboud I, Muyo G, Gorman A, Sallam A, Rodmell $S$ et al. Validation of human whole blood oximetry using a hyperspectral camera with a model eye. Invest Ophthalmol Vis Sci 2011; e-pub ahead of print 10 January 2011.

41 Gorman A, Fletcher-Holmes DW, Harvey AR. Harvey, generalisation of the Lyot filter and its application to snapshot spectral imaging. Opt Express 2010; 18(6): 5602-5608. 